\title{
Early Onset Collagen VI Myopathies: Genetic and Clinical Correlations
}

Laura Briñas, PhD, ${ }^{1,2}$ Pascale Richard, PhD, 1,2,3 Susana Quijano-Roy, MD, PhD, 1,2,4

Corine Gartioux, BS, ${ }^{1,2}$ Céline Ledeuil, BS, ${ }^{3}$ Emmanuelle Lacène, BS, ${ }^{1,2}$

Samira Makri, MD, ${ }^{5}$ Ana Ferreiro, MD, PhD, 1,2,4,6 Svetlana Maugenre, BS, 1,2

Haluk Topaloglu, MD, ${ }^{7}$ Göknur Haliloglu, MD, ${ }^{7}$ Isabelle Pénisson-Besnier, MD, ${ }^{8}$

Pierre-Yves Jeannet, MD, ${ }^{9}$ Luciano Merlini, MD, ${ }^{10,11}$ Carmen Navarro, MD, PhD, ${ }^{12}$

Annick Toutain, MD, PhD, ${ }^{13}$ Denys Chaigne, MD, ${ }^{14}$ Isabelle Desguerre, MD, ${ }^{15,16}$

Christine de Die-Smulders, MD, ${ }^{17}$ Murielle Dunand, MD, ${ }^{18}$ Bernard Echenne, MD, PhD, ${ }^{19}$

Bruno Eymard, MD, PhD, ${ }^{6}$ Thierry Kuntzer, MD, ${ }^{18}$ Kim Maincent, MD, ${ }^{20}$

Michèle Mayer, MD, ${ }^{20}$ Ghislaine Plessis, MD, ${ }^{21}$ François Rivier, MD, PhD, ${ }^{19}$

Filip Roelens, MD, ${ }^{22}$ Tanya Stojkovic, MD, ${ }^{6}$ Ana Lía Taratuto, MD, PhD, ${ }^{23}$

Fabiana Lubieniecki, MD, ${ }^{24}$ Soledad Monges, MD, ${ }^{25}$ Christine Tranchant, MD, ${ }^{26}$ Louis Viollet, MD, PhD, ${ }^{4,27}$ Norma B. Romero, MD, PhD, ${ }^{1,2}$ Brigitte Estournet, MD, PhD, ${ }^{4}$ Pascale Guicheney, $\mathrm{PhD},{ }^{1,2,3}$ and Valérie Allamand, $\mathrm{PhD}^{1,2}$

View this article online at wileyonlinelibrary.com. DOI: 10.1002/ana.22087

Received Jun 22, 2009, and in revised form Apr 30, 2010. Accepted for publication May 17, 2010

Address correspondence to Dr Allamand, UMRS 974, Institut de Myologie, Bâtiment Babinski, GH Pitié-Salpêtrière, 47 boulevard de I’Hôpital, 75651

Paris cedex 13, France. E-mail: v.allamand@institut-myologie.org

Current address for L.B., S.Q.-R., C.G., T.S., N.B.R., and V.A. UPMC University Paris 06, Institut de Myologie, Inserm, U974 and CNRS, UMR7215,

Groupe Hospitalier Pitié-Salpêtrière, Paris, France.

Current address for P.R., S. Maugenre, and P.G. Inserm U956, CHU Pitié Salpêtrière, Paris, France.

Current address for A.F. Inserm, U787-Institut de Myologie, Groupe Hospitalier Pitié-Salpêtrière, Paris, France.

From the ${ }^{1}$ Inserm, U582, Paris, France; ${ }^{2}$ UPMC University of Paris 06, IFR14, Myology Institute, Paris, France; ${ }^{3}$ AP-HP, Pitie -Salpêtrière Hospital Group, Cardiogenetics and Myogenetics Functional Unit, Metabolic Biochemistry Division, Paris, France; ${ }^{4}$ Assistance Publique-Hôpitaux de Paris, Pediatric Division,

Neuromuscular Disease Reference Center, Raymond Poincaré Hospital, Garches, France; ${ }^{5}$ Neurology Division, Ali Ait Idir Specialized Hospital, Alger,

Algeria; ${ }^{6}$ Paris-Est Neuromuscular Disease Referal Centre, Myology Institute, Pitié-Salpêtrière Hospital Group, Paris, France; ${ }^{7}$ Section of Neurology,

Department of Pediatrics, Hacettepe Children's Hospital, Ankara, Turkey; ${ }^{8}$ Neuromuscular Disease Reference Center, Department of Neurology, University Hospital, Angers, France; ${ }^{9}$ Neuropediatry Division, CHUV-BH11, Lausanne, Switzerland; ${ }^{10}$ Department of Experimental and Diagnostic Medicine, Section of Medical Genetics, University of Ferrara, Ferrara, Italy; ${ }^{11}$ Laboratory of Musculoskeletal Cell Biology, Rizzoli Orthopedic Institute, Bologna, Italy;

${ }^{12}$ Department of Pathological Anatomy, Hospital Meixoeiro, Vigo University Hospital, Vigo, Spain; ${ }^{13}$ Genetics Division, Bretonneau University Hospital,

Tours, France; ${ }^{14}$ Cliniq Sainte-Odile, Strasbourg, France; ${ }^{15}$ Assistance Publique-Hôpitaux de Paris, Department of Neuropediatrics, Neuromuscular Disease Reference Center "Garches-Necker-Mondor-Hendaye," Necker-Enfants Malades Hospital, Paris, France; ${ }^{16}$ Department of Neurosciences, Team 10 Inserm, U841 Mondor Biomedical Research Institute, Paris XII University, Créteil, France; ${ }^{17}$ Department of Clinical Genetics, University Hospital Maastricht,

Maastricht, the Netherlands; ${ }^{18}$ Nerve-Muscle Unit, Neurology Division, BH07/309, University Hospital Vaudois, Lausanne, Switzerland; ${ }^{9}$ University Hospital of Montpellier, Neuropediatry Division-Grand Sud Ouest Neuromuscular Disease Reference Center, Gui de Chauliac Hospital, Montpellier, France;

${ }^{20}$ Armand Trousseau Hospital, Neuropediatry Division, Neuromuscular Disease Center, Paris, France; ${ }^{21}$ Medical Genetics Division, DGR, Clémenceau

University Hospital, Caen, France; ${ }^{22}$ Dominiek Savio Instituut Gits, Roeselare, Belgium; ${ }^{23}$ Department of Neuropathology/FLENI, Institute for Neurological Research, Buenos Aires, Argentina; ${ }^{24}$ Department of Pathology, Garrahan National Pediatric Hospital, Buenos Aires, Argentina; ${ }^{25}$ Department of

Neuropediatrics, Garrahan National Pediatric Hospital, Buenos Aires, Argentina; ${ }^{26}$ Neurology Division, Strasbourg University Hospitals, Strasbourg, France; ${ }^{27}$ Inserm, U781, Necker-Enfants Malades Hospital, Paris, France. 
Objective: Mutations in the genes encoding the extracellular matrix protein collagen VI (ColVI) cause a spectrum of disorders with variable inheritance including Ullrich congenital muscular dystrophy, Bethlem myopathy, and intermediate phenotypes. We extensively characterized, at the clinical, cellular, and molecular levels, 49 patients with onset in the first 2 years of life to investigate genotype-phenotype correlations.

Methods: Patients were classified into 3 groups: early-severe (18\%), moderate-progressive (53\%), and mild (29\%). ColVI secretion was analyzed in patient-derived skin fibroblasts. Chain-specific transcript levels were quantified by quantitative reverse transcriptase polymerase chain reaction (qRT-PCR), and mutation identification was performed by sequencing of complementary DNA.

Results: ColVI secretion was altered in all fibroblast cultures studied. We identified 56 mutations, mostly novel and private. Dominant de novo mutations were detected in $61 \%$ of the cases. Importantly, mutations causing premature termination codons (PTCs) or in-frame insertions strikingly destabilized the corresponding transcripts. Homozygous PTC-causing mutations in the triple helix domains led to the most severe phenotypes (ambulation never achieved), whereas dominant de novo in-frame exon skipping and glycine missense mutations were identified in patients of the moderate-progressive group (loss of ambulation).

Interpretation: This work emphasizes that the diagnosis of early onset ColVI myopathies is arduous and timeconsuming, and demonstrates that quantitative RT-PCR is a helpful tool for the identification of some mutationbearing genes. Moreover, the clinical classification proposed allowed genotype-phenotype relationships to be explored, and may be useful in the design of future clinical trials.

ANN NEUROL 2010;68:511-520

$T_{\mathrm{vi}}^{\text {he }}$ e ubiquitous extracellular matrix protein collagen VI (ColVI) is composed of 3 chains, $\alpha 1(\mathrm{VI}), \alpha 2(\mathrm{VI})$, and $\alpha 3(\mathrm{VI})$, which display a similar structure with a triple helical (TH) domain, characterized by the repetition of the Gly-X-Y amino acid sequence, and flanked by globular domains homologous to von Willebrand factor A domains. ${ }^{1,2}$ These chains assemble in tetramers before secretion into the extracellular matrix to form microfibrils of ColvI. ${ }^{3}$

Mutations in the COLGA1, COL6A2, and COLGA3 genes cause ColVI myopathies such as Ullrich congenital muscular dystrophy (UCMD, Mendelian Inheritance in Man [MIM]\# 254090) and Bethlem myopathy (BM, MIM\# 158810), 2 entities that constitute the extremities of a continuous clinical spectrum, ${ }^{4,5}$ with intermediate phenotypes also observed. Classical UCMD, the severe and progressive form, presents with hypotonia, muscle weakness, and motor delay in the first months of life and is characteristically associated with proximal joint contractures and a striking distal hyperlaxity, as well as severe respiratory insufficiency. ${ }^{6,7}$ Classical BM is usually of later onset and corresponds to a milder phenotype with slow or static course, characterized by elbow and long finger flexor contractures. ${ }^{8,9}$ In addition, mutations in the ColVI-encoding genes have been associated with 2 other neuromuscular disorders: autosomal dominant limb-girdle muscular dystrophy in 3 families and, more recently, autosomal recessive myosclerosis myopathy (MIM\# 255600) in 1 family. ${ }^{10,11}$ Moreover, although UCMD was initially described as an autosomal recessive disorder, autosomal dominant mutations have been increasingly reported. ${ }^{12-22}$ On the other hand, although $\mathrm{BM}$ is mostly inherited as an autosomal dominant disease, rare de novo mutations have been published, ${ }^{23}$ and
2 recent studies report autosomal recessive mutations. $^{24,25}$ As a consequence of this great heterogeneity at the clinical and molecular levels, achieving a definite diagnosis is arduous and cumbersome, and establishing genotype-phenotype correlations has proven a difficult task.

Here we present a large cohort of 49 patients with early onset ColVI myopathy with a variable severity of presentation and progression, and harboring mutations in 1 of the ColVI-encoding genes. The vast majority of the mutations were novel, and more than half of them were dominant de novo. Quantitative reverse transcriptase polymerase chain reaction (qRT-PCR) proved useful to identify the mutation-bearing gene in the case of inframe insertions and mutations leading to premature termination codons (PTCs), which greatly reduce the stability of the corresponding transcripts. Overall, this study revealed several genotype-phenotype correlations, providing new insights into the natural history and course of ColVI myopathies.

\section{Subjects and Methods \\ Patients}

We studied 49 unrelated index patients (24 males; 25 females) from different origins (Algeria, Argentina, Belgium, France, Italy, Madagascar, Portugal, Spain, Switzerland, the Netherlands, and Turkey). All of them presented with weakness, serum creatine kinase levels no more than $4 \times$ normal values, contractures, and/or distal hyperlaxity. All cases had onset of symptoms in infancy, and their parents showed no clinical involvement. Their ages at last examination ranged from 4 to 32 years (mean age, 16 years). Three patients from consanguineous parents had affected siblings, whereas the others were sporadic cases. Signed informed consents were obtained for genetic analysis according to the French legislation on ethical rules. 
TABLE: Summary of the Clinical, Genetic, and Immunolabeling Data in Each Group of Patients

\begin{tabular}{|c|c|c|c|c|c|c|}
\hline \multirow[b]{2}{*}{$\begin{array}{l}\text { Clinical } \\
\text { Group }\end{array}$} & \multirow{2}{*}{$\begin{array}{l}\text { Number of } \\
\text { Patients } \\
\text { (male/ } \\
\text { female) }\end{array}$} & \multirow{2}{*}{$\begin{array}{l}\text { Range of Age } \\
\text { (mean) at Last } \\
\text { Examination, } \\
\text { yr }\end{array}$} & \multirow[b]{2}{*}{$\begin{array}{l}\text { Walked } \\
\text { before } \\
24 \text { Months, \% }\end{array}$} & \multirow{2}{*}{$\begin{array}{l}\text { Mean Age } \\
\text { at Loss of } \\
\text { Ambulation, } \\
\text { yr }\end{array}$} & \multicolumn{2}{|c|}{ Congenital } \\
\hline & & & & & Contractures, \% & $\begin{array}{l}\text { Hip } \\
\text { Dislocation, \% }\end{array}$ \\
\hline $\begin{array}{l}\text { Early } \\
\text { severe }^{d}\end{array}$ & $9(7 / 2)$ & $4-15(10)$ & Never walked & - & 89 & 56 \\
\hline $\begin{array}{l}\text { Moderate } \\
\text { progressive }^{\mathrm{e}}\end{array}$ & $26(12 / 14)$ & $5-37(16.4)$ & 61.5 & 10 & 54 & 58 \\
\hline Mild & $14(5 / 9)$ & $5-32(20)$ & 93 & $\begin{array}{l}\text { Still } \\
\text { ambulant }\end{array}$ & 57 & $>64$ \\
\hline
\end{tabular}

${ }^{\mathrm{a}} \mathrm{FVC}=$ forced vital capacity at last examination. Mean values were calculated from available data (8 early-severe, 20 moderate-progressive, and 8 mild patients).

${ }^{\mathrm{b}} \mathrm{PTC}=$ premature termination codon; Ex skip = exon skipping (in frame); IF del/ins = in-frame deletion or insertion.

${ }^{\mathrm{c}} \mathrm{H}=$ homozygote; $\mathrm{hn}=$ heterozygote de novo; $\mathrm{ch}=$ compound heterozygote; $\mathrm{uk}=$ unknown.

${ }^{\mathrm{d}}$ Two patients deceased.

${ }^{\mathrm{e}}$ One patient deceased.

ColVI = collagen VI.

\section{Collagen VI Immunolabeling}

Muscle cryosections were available for 33 patients and were colabeled using monoclonal antibodies against human ColVI (MAB1944 or MAB3303, Chemicon International, Temecula, CA) diluted 1:200, and against human perlecan (Chemicon International) diluted 1:100. Sections were observed with a Nikon (Melville, NY) E 600 fluorescence microscope.

ColVI immunolabeling in skin fibroblasts from 4 controls and 47 patients was carried out as previously reported. ${ }^{26}$ Fibroblast cultures from the remaining 2 patients were not available or did not grow properly and could not be analyzed. Briefly, confluent cells were cultured in the presence of $0.25 \mathrm{mM}$ ascorbic acid for 5 days and stained with the MAB1944 antibody (Chemicon International), diluted 1:100. Slides were observed with an Axioplan 2 microscope (Zeiss, Munich, Germany) equipped with a HBO 100 mercury lamp (Zeiss), and representative images were obtained using the Metaview software (Roper Scientific, Trenton, NJ).

\section{RNA Extraction and Reverse Transcription}

Total RNA was extracted from confluent fibroblast cultures using Trizol (Invitrogen, Carlsbad, CA) following the manufacturer's instructions. cDNA was prepared from $1 \mu \mathrm{g}$ of total RNA using oligo $(\mathrm{dT})_{18}$ and Superscript II Reverse Transcriptase as recommended by the manufacturer (Invitrogen).

\section{Quantitative RT-PCR}

Chain specific transcripts were quantified from fibroblastderived cDNA using quantitative RT-PCR on a LightCycler 480 apparatus (Roche Diagnostics, Mannheim, Germany), using primers hybridizing to the last $\mathrm{C}$-terminal domain of each chain, and $\beta$-actin as a reference (Supporting Information Table S1). The $2^{-\Delta \Delta \mathrm{CT}}$ method was used to determine the rela- tive concentrations. ${ }^{27}$ Quantitative RT-PCR results are expressed as the mean value of the different experiments performed on at least 2 different cDNA samples for each individual. Significant differences were determined performing Student $t$ test $(p<0.05)$.

\section{Genetic Analysis}

Genomic DNA from patients and their relatives was obtained from blood leucocytes by standard methods. Genetic results of 7 patients were previously reported. ${ }^{26,28}$ Additional consanguineous and informative nonconsanguineous families were genotyped with flanking and intragenic microsatellite markers corresponding to the COL6A1/COL6A2 (D21S156, D21S171, HCOLINT1, HCOLINT2, D21S2058) and COL6A3 (D2S338, D2S345, D2S2968, HCOLINT, D2S2253) loci to study the transmission of the parental haplotypes. ${ }^{28}$

The coding regions of the genes were amplified from fibroblast-derived cDNA in several overlapping fragments (primers available on request), and sequenced on the ABI3730 capillary electrophoresis system (Applied Biosystems, Foster City, $\mathrm{CA})$. Sequences were compared to reference sequences NM_001848.2 (COL6A1), NM_001849.3 (COL6A2), and NM_004369.3 (COL6A3) of the National Center for Biotechnology Information database. Each potentially pathogenic change was confirmed on genomic DNA from patients and their relatives. Nonsynonymous amino acid substitutions were considered as possibly pathogenic mutations when they affected a highly conserved residue suspected to play an important role in the function of the protein and/or when they were not detected in 100 unrelated control individuals. Alamut (Interactive Biosoftware, North Seattle, WA) and Polyphen (http://coot.embl.de/PolyPhen) softwares were also used to determine the pathogenicity of the variants. 


\begin{tabular}{|c|c|c|c|c|c|c|c|c|}
\hline \multirow{2}{*}{$\begin{array}{l}\text { Mean } \\
\text { FVC, \% } \\
\text { (range) }^{\mathrm{a}}\end{array}$} & \multirow{2}{*}{$\begin{array}{l}\text { Spinal } \\
\text { deformities, } \\
\%\end{array}$} & \multicolumn{2}{|c|}{ ColVI Secretion } & \multicolumn{3}{|c|}{ Mutated Gene, \% } & \multirow{2}{*}{$\begin{array}{l}\text { Predicted } \\
\text { Consequence } \\
\%^{\mathrm{b}}\end{array}$} & \multirow[b]{2}{*}{$\begin{array}{l}\text { Inheritance } \\
\%^{\mathrm{c}}\end{array}$} \\
\hline & & $\begin{array}{l}\text { Muscle } \\
\%\end{array}$ & $\begin{array}{l}\text { Fibroblast } \\
\%\end{array}$ & COLGA1 & COLGA2 & COLGA3 & & \\
\hline $30(15-46)$ & 100 & $\begin{array}{l}\text { Abnormal } \\
(100)\end{array}$ & $\begin{array}{l}\text { Abnormal } \\
(100)\end{array}$ & 44.5 & 11 & 44.5 & $\begin{array}{l}\text { PTC (67), } \\
\text { Missense (11), } \\
\text { Ex skip (22) }\end{array}$ & $\begin{array}{l}\mathrm{H}(67) \\
\text { hn }(33)\end{array}$ \\
\hline $39.6(15-70)$ & 100 & $\begin{array}{l}\text { Abnormal } \\
(76.5)\end{array}$ & $\begin{array}{l}\text { Abnormal } \\
(100)\end{array}$ & 28 & 55 & 17 & $\begin{array}{l}\text { PTC (14), } \\
\text { Missense (45), } \\
\text { Ex skip (38), } \\
\text { IF del/ins (3) }\end{array}$ & $\begin{array}{l}\mathrm{H}(4), \\
\text { hn }(85), \\
\text { ch }(11)\end{array}$ \\
\hline $63(35-82)$ & 86 & $\begin{array}{l}\text { Abnormal } \\
(50)\end{array}$ & $\begin{array}{l}\text { Abnormal } \\
(100)\end{array}$ & 28 & 50 & 22 & $\begin{array}{l}\text { PTC (33), } \\
\text { Missense (44), } \\
\text { Ex skip (6), } \\
\text { IF del/ins (17) }\end{array}$ & $\begin{array}{l}\text { H }(28.5), \\
\text { hn }(36), \\
\text { ch }(28.5), \\
\text { uk }(7)\end{array}$ \\
\hline
\end{tabular}

\section{Results}

\section{Patient Phenotypes and Clinical Classification}

A great heterogeneity in the severity and course of the disease was observed among the 49 patients, although all of them had initial symptoms in the first 2 years of life. Because of this heterogeneity, and because most of the patients had long-term follow-up, they were divided into 3 groups according to their maximal motor capacity and their progression, as proposed by Quijano Roy et $\mathrm{al}^{4,29}$ : the most severe group (early-severe) included 9 patients (18\%) who never achieved ambulation. The moderateprogressive group was composed of 26 patients (53\%) who were initially able to walk but showed a very progressive course during childhood or early adulthood. Indeed, loss of ambulation occurred at a mean age of 10 years (age range, 4-25 years), and only 3 patients above that age were still able to walk indoors at their last examination (mean age, 19 years). The less affected group (mild) included 14 patients (29\%) who remained ambulatory (mean age of 20 years at last examination). Clinical data are summarized in the Table and detailed in Supporting Information Table S2.

All patients in the early-severe group presented with axial or proximal deformities at birth such as torticollis, kyphoscoliosis, or shoulder, elbow, hip, or knee joint contractures. These findings were also observed in 9 patients from the moderate-progressive group (35\%) and 3 from the mild group (21\%). Two ambulatory children classified at 5 and 9 years in the mild group had antecedents of torticollis or proximal joint contractures, but due to their young ages it is difficult to exclude that they will not progress to the moderate group. Restrictive respiratory progressive insufficiency was very frequent, and 25 patients (including all those from the early-severe group) had forced vital capacity $<50 \%$ of theoretical values. Thirty-four percent of the patients in the early-severe and moderate-progressive groups were mechanically ventilated $(16 \%$ by nasal nocturnal ventilation and $18 \%$ via tracheotomy), and 3 patients died in their teens possibly due to respiratory complications (see Supporting Information Table S2). Spinal involvement (stiffness, with or without deformity) was observed at birth (4 cases) or in the course of the disease ( 43 patients), consisting usually in scoliosis (31 patients), associated with kyphosis in 16 of them. The remaining patients showed isolated dorsal kyphosis, lumbar lordosis, or only spinal stiffness without significant deformity. Cutaneous abnormalities such as granular rough skin or hypertrophic scars were noted in 22 patients (45\%) from the 3 groups, but this may be underestimated.

\section{ColVI Expression and Secretion Pattern in Cultured Skin Fibroblasts and Muscle Biopsies}

Control fibroblasts abundantly expressed and secreted ColVI, which formed a dense network (Fig 1). In contrast, an abnormal secretion was detected in all the patient fibroblast cultures (see Table and Supporting Information Table S2). ColVI secretion was either completely absent (23 samples, 52\%), strongly reduced (10 patients, 23\%), or slightly reduced (11 cases, 25\%). In these 3 groups, an intracellular retention was frequently observed $(83 \%)$. In some cases with reduced $(n=5)$ or 
strongly reduced $(\mathrm{n}=3)$ secretion, a dotted pattern was observed. In the 3 remaining patients, the results were not interpretable.

In contrast, ColVI immunolabeling on muscle sections was identical to controls in $27 \%$ of the patients (see Supporting Information Table S2). An intracellular staining was observed in muscle fibers of $18 \%$ of the patients, a peculiar finding that remains to be investigated further.

\section{Mutation Identification in the COL6A Genes}

Sequencing of fibroblast-derived cDNA enabled the identification of 56 different mutations (on 67 alleles), of which $73 \%$ had not been previously reported (Fig 2 and see Supporting Information Table S2). These mutations were quite evenly distributed in the 3 genes: 17 in COLGA1 (30\%), 26 in COLGA2 (46\%), and 13 in COL6A3 (23\%) (see Fig 2). In 7 consanguineous families, homozygosity mapping was useful to direct the sequencing effort. ${ }^{26,28}$

A total of 30 patients (61\%) harbored dominant de novo mutations (Supporting Information Tables S2 and S3). In 1 patient, a single heterozygous mutation was identified, but no conclusion on its inheritance could be established for lack of the parents' DNA. The 18 remaining patients carried recessive mutations, 11 in a homozygous state (22\%) and 7 as compound heterozygotes $(14 \%)$. It is worth mentioning that only 2 unrelated patients share the same genomic mutation (see Supporting Information Table S2).

All the mutations in COL6A1, as well as all the de novo mutations in COLGA2 and COLGA3, were located within the TH domains of the chains. In addition, exon 10 of COL6A1, exon 26 of COL6A2, and intron 16 of COL6A3 were preferentially mutated $(38 \%, 10 \%$, and $18 \%$ of the mutated alleles in each gene, respectively).

PTC-causing mutations were identified in 14 patients (28.5\%), including 9 homozygotes and 5 compound heterozygotes, and represented $37 \%$ of the mutated alleles. In 8 of the homozygous patients, the changes were located upstream or within the TH domain, whereas the remaining 1 harbored a large homozygous deletion corresponding to $8 \mathrm{~kb}$ of genomic DNA downstream of exon 26 of COL6A2, leading to the loss of the $\mathrm{C} 2$ domain. Two of the 5 compound heterozygous patients carried 2 PTC-causing mutations located in the $\mathrm{C} 1$ domain of the $\alpha 2(\mathrm{VI})$ chain, and the other 3 patients combined a PTC-causing mutation, upstream or downstream of the TH domain of $\alpha 2(\mathrm{VI})$, with a missense mutation or with an in-frame insertion (see Supporting Information Table S2).
Missense mutations were identified in 20 patients $(41 \%)$ and represented $33 \%$ of the mutated alleles. In $75 \%$ of these patients, the mutations involved a glycine residue of the repeated Gly-X-Y motif of the TH domains, and were dominant de novo. The remaining 25\% were either homozygote (1 patient), missense compound heterozygote (1 patient), or combined a missense mutation with a PTCcausing mutation (2 patients) or an in-frame deletion (1 patient; see Supporting Information Table S2).

Several exon skipping events due to dominant de novo splice-site mutations were detected in 14 patients (28.5\%), representing $21 \%$ of the mutated alleles. Notably, several patients harbored different genomic mutations leading to identical consequences both at the mRNA and protein levels. For example, the skipping of exon 5 in COL6A2 was due to either donor (c.801 G>A and c.801+2 $\mathrm{T}>\mathrm{C}$ ) or acceptor (c.736-3 C>G) splicesite mutations. Similarly, skipping of exon 16 of COL6A3 was caused by 3 different changes affecting the same donor site (c.6210+1 G>A, c.6210+1 G>T, and c. $6210+5 \mathrm{G}>\mathrm{A}$ ), and skipping of exon 18 of COL6A3 was due to either c.6309+1 G>A or c.6309+3 A>G mutations (see Supporting Information Table S2).

Finally, 4 patients harbored small in-frame deletions, affecting 1 or 3 amino acids (1 patient in COL6A1, another in COL6A3), or an insertion of 3 amino acids in COL6A2 (2 patients).

Additionally, a large number of potentially nonpathogenic variants were also identified, emphasizing the highly polymorphic nature of these genes (Supporting Information Table S4).

\section{Quantification of Chain-Specific Transcripts}

We developed a chain-specific quantitative RT-PCR assay and determined the transcript levels of each chain for 48 patients. Results were normalized to a control used as calibrator. Transcripts extracted from fibroblasts from 5 additional control individuals were analyzed and showed some variability, with levels ranging from 0.58 to 2.21 for COL6A1 (mean value, $1.49 \pm 0.59$ ), 0.59 to 1.14 for COL6A2 (mean value, $0.95 \pm 0.13$ ), and 0.64 to 1.13 for COL6A3 (mean value, $0.77 \pm 0.24$ ). Patients transcripts levels ranged from 0.03 to 2.43 for COL6AI (mean value, $0.53 \pm 0.44$ ), 0.06 to 1.07 for COL6A2 (mean value, $0.35 \pm 0.23$ ), and 0.03 to 1.3 for COL6A3 (mean value, $0.45 \pm 0.29$ ). Notably, in 13 of the patients $(26.5 \%)$ the levels of only 1 transcript were $\leq 0.10$ and corresponded to the mutated chain. In $85 \%$ of these cases, the mutation induced either PTCs or inframe insertions. In contrast, in the other 35 patients, all 3 chain transcripts showed levels $>0.10$ and corresponded to patients bearing in-frame deletions or 

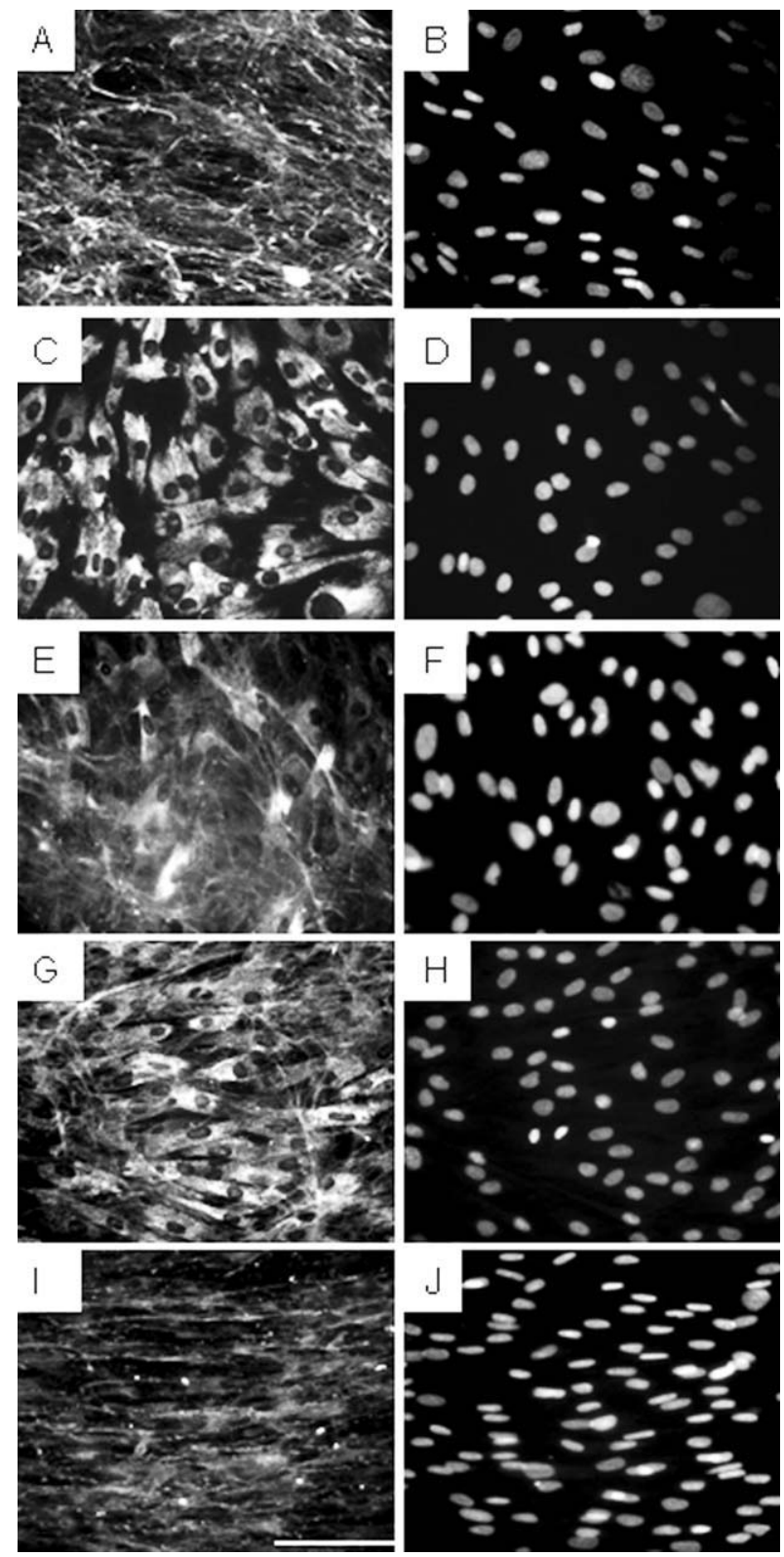

FIGURE 1: Collagen VI (ColVI) immunolabeling of controland patient-derived fibroblasts in culture. (A) Control fibroblasts forming a dense ColVI network; (C, E, G, I) ColVI immunolabeling in fibroblasts from patients as examples of complete secretion deficiency, strongly reduced secretion, reduced secretion, and dotted pattern, respectively. Intracellular retention of ColVI can be observed in all these cases. (B, D, F, H, J) Nuclei were labeled with 4,6-diamidino2-phenylindole to control cell density of the cultures. Bar corresponds to $100 \mu \mathrm{m}$.

missense mutations, combined in 2 cases with PTC-causing mutations. Mean values of the mRNA levels of the mutated chains according to the type of mutation are presented in Figure 3 (individual values are presented in Supplementary Fig S1). The levels of transcripts harboring heteroallelic PTC-causing mutations as well as inframe insertions were consistently strikingly diminished, ranging from 0.03 to 0.10 , except for 1 patient $(0.19)$, whereas those with missense mutations and in-frame deletions were less reduced or close to normal (0.11$0.99)$, except for 3 patients $(0.10,0.06$, and 2.43$)$.

To rule out transcriptional inhibition, the levels of pre-mRNA harboring PTC-causing mutations and inframe insertions were quantified and were not found different from a control sample, suggesting that the degradation of transcripts harboring these mutations occurred at the post-transcriptional level (data not shown).

\section{Correlations with the Clinical Phenotype}

Taking together all the results presented above, we set out to establish correlations between the clinical severity and (1) ColVI secretion in cultured fibroblasts and muscle sections and (2) genotype.

With respect to the degree of abnormality in the secretion of ColVI in cultured fibroblasts, all patients from the early-severe group and $83 \%$ of the patients classified as moderate-progressive displayed a complete absence or a strongly reduced secretion, as opposed to $52 \%$ of the mildly affected patients. In addition, abnormal ColVI secretion was observed in the muscle section from all the patients of the early-severe group (see Table and Supporting Information Table S2).

Genotype-phenotype correlations could be established from this study; homozygous PTC-causing mutations (before or within the TH domains) were associated with the most severe clinical phenotypes (6 patients of 9 in the early-severe group). In contrast, 2 patients carrying homozygous PTC-causing mutations in the $\mathrm{N}$-terminal domains displayed a mild clinical phenotype. Additionally, $77 \%$ of the patients bearing de novo mutations that lead to the skipping of exons encoding part of the $\mathrm{TH}$ domains, as well as $66 \%$ patients with de novo missense mutations affecting a glycine residue of the TH Gly-X-Y motif, were identified in the moderate-progressive group.

Finally, although de novo dominant mutations were most common overall, recessive inheritance was found in all 3 clinical categories.

\section{Discussion}

Numerous reports have shown the important clinical variability of UCMD and the complexity of the molecular diagnosis, with either recessive or dominant de novo mutations, ${ }^{5,14-16,18,21,30}$ and possible incomplete penetrance. ${ }^{21}$ Here, we report a cohort of 49 unrelated patients with early onset ColVI myopathy, which we characterized at the clinical, cellular, and molecular levels to establish genotype-phenotype correlations. Although most of the patients in this series may be included in the UCMD 


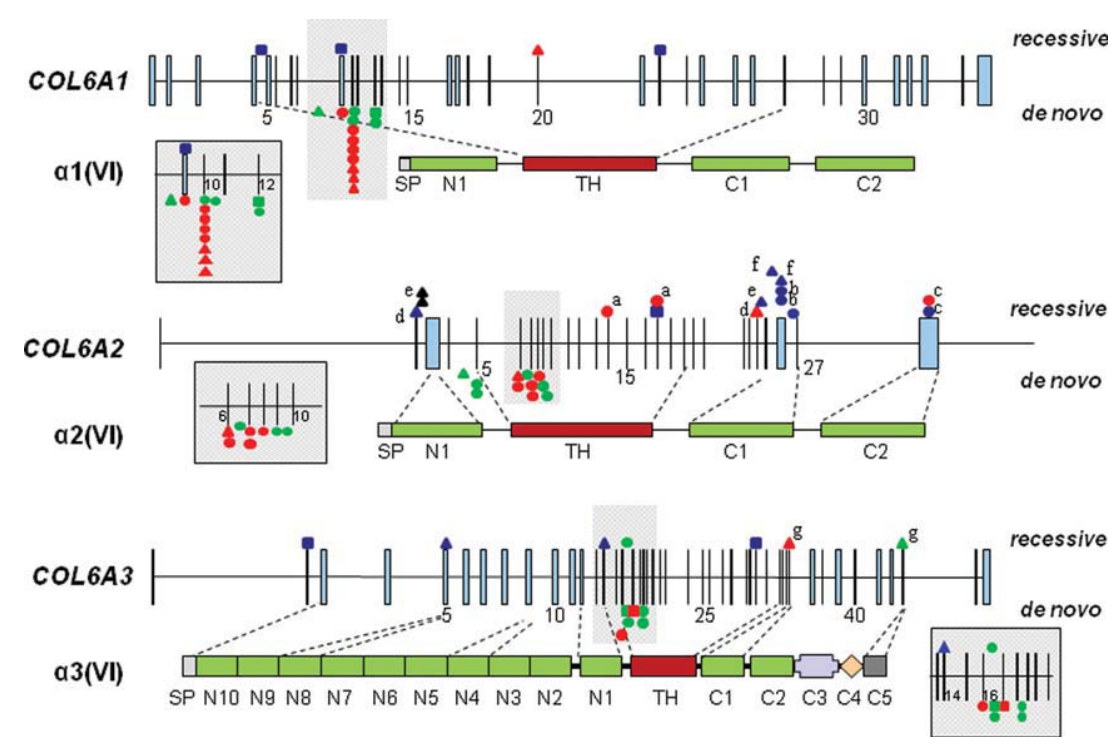

FIGURE 2: Schematic representation of mutations identified in the COL6A1, COL6A2, and COL6A3 genes. Exons are shown as blue vertical rectangles above the corresponding protein. The signal peptide (SP) and the domains are indicated below the diagram (TH = triple helical domain; N10-N1 = N-terminal domains; C1-C5 = C-terminal domains). Recessive and dominant de novo mutations are indicated above or below the genes, respectively. Premature termination codon-causing mutations are indicated in blue, missense mutations in red, and in-frame deletions and insertions in green and black, respectively. Symbols refer to the clinical phenotype of the patients: early-severe as squares, moderate-progressive as circles, and mild as triangles. Letters a through $\mathrm{g}$ indicate that patients are compound heterozygotes and refer to the 2 mutations identified in the same patient. Shaded areas containing several mutations are enlarged.

category, we prefer to use the term "early onset" ColVI myopathy because of the existence of patients with an early onset (before 24 months) but a slowly progressive course (mild patients) who do not present typical characteristics either of UCMD or of BM, and who could belong to an intermediate group. This overlap has been also previously stressed by others in patients with later onset (more similar to typical BM) and a more progressive course than expected (severe or progressive BM). ${ }^{4,31}$

Clinically, patients showed a large spectrum of severity, with variable impairment of motor and respiratory function. We chose to classify them according to their maximal motor capacity rather than respiratory function due to the variability in respiratory management between centers, and the close association between mobility loss and need for respiratory support. ${ }^{32}$ Therefore, 3 motor severity groups were established, and distinguished patients who never achieved ambulation, walked but lost this ability (or were about to lose it), or were still fully ambulatory. This classification was useful to infer genotype-phenotype correlations in nonambulatory patients, and in those ambulatory with a long follow-up. However, molecular data regarding young ambulatory children should be considered carefully, because they may or may not follow a progressive and severe course later on. We tried to investigate early signs of severity or predictors of progression suggested in a previous study, ${ }^{29}$ and found that all nonambulatory patients and $1 / 3$ of moderate-progressive patients had shown proximal and/or axial orthopedic deformities at birth. However, this sign was also found in 3 patients still ambulatory in the second or third decade, meaning that some patients with congenital

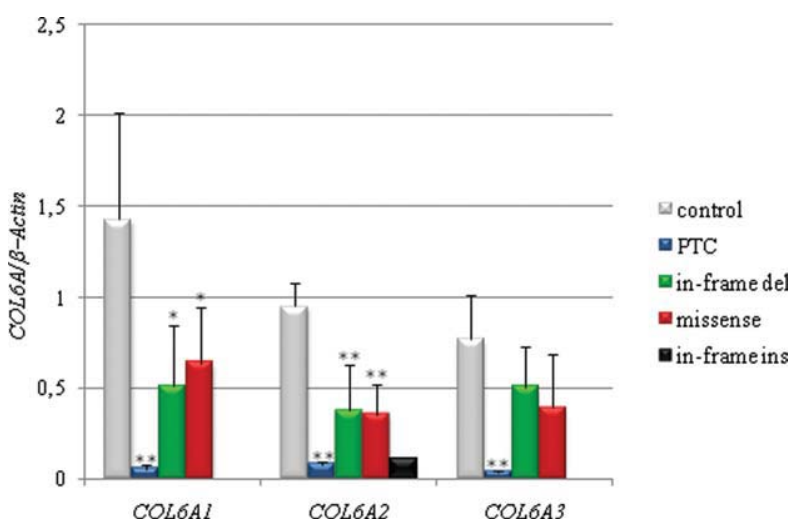

FIGURE 3: COL6A transcript levels from control- and patient-derived fibroblasts. Only patients harboring a single type of mutation were taken into account. Transcript levels of the mutated chain were classified according to the type of mutation identified. Statistical analysis could not be carried out in samples harboring COL6A2 in-frame insertion because only 1 homozygous patient was identified. Data are presented as mean values \pm standard deviation $\left({ }^{* *} p<\right.$ $0.01,{ }^{*} p<0.02$ ). PTC $=$ premature termination codon; del $=$ deletion; ins = insertion. [Color figure can be viewed in the online issue, which is available at wileyonlinelibrary.com.] 
contractures or deformities of the proximal joints, neck, or spine may have a relatively good prognosis. Finally, in our series, respiratory function was more severely impaired in the oldest patients of both the early-severe and moderateprogressive groups, but not in the mild patients.

At the molecular level, mutations were largely private (only 1 mutation detected in 2 unrelated patients), and the vast majority of them are novel. The cDNAbased strategy, unveiling the consequences of a genomic mutation, allowed us to identify mutations that would have been difficult to detect at the genomic level, such as the intronic mutation c.904-39 A>G affecting a branch point and leading to COL6A1 exon 11 skipping. We also identified the largest genomic deletion in the COL6A2 gene so far. Importantly, $61 \%$ of the mutations were dominant de novo, in accordance to previous studies, ${ }^{16}$ which is a limitation for linkage analysis approaches. We report 5 dominant mutations already identified as de novo, ${ }^{15,16,18,21}$ indicating that some regions of the COL6A genes could be more susceptible to neomutations. Most of the mutations affecting the Gly-X-Y repetitions were located in the $\mathrm{N}$-terminal part of the $\mathrm{TH}$ domains, emphasizing the importance of this region. Indeed, it has been demonstrated by in vitro studies that such mutations are compatible with the integration of the mutated chain in the tetramer, leading to reduced microfibril formation efficiency, ultimately exerting a dominant deleterious effect. ${ }^{33}$ In contrast with other reports, ${ }^{14,18}$ we observed that most of the patients at the severe end of the clinical spectrum (early-severe and moderate-progressive phenotypes) display a striking reduction or complete absence of ColVI secretion, as opposed to only half of the least affected patients. According to our results, the association of clinical symptoms and a striking anomaly of ColVI secretion could be predictive of a more severe course.

A cellular mechanism that regulates the expression of ColVI is the degradation of abnormal transcripts. Indeed, we showed that all types of mutations can lead to reduced transcript levels. Moreover, quantitative analysis of pre-mRNA levels suggested that this regulation does not occur at the transcriptional level (data not shown), but that these mutations destabilize the corresponding mRNA molecules, most strikingly in the case of PTC-causing mutations and in-frame insertions. Reduced transcripts levels were also reported in 4 patients harboring splicing mutations leading to either exon skipping or PTCs. ${ }^{34}$ It is worth noting that, in the case of PTC-causing mutations as well as in-frame insertions, the quantification of transcript levels allowed us to detect the mutated gene prior to sequencing. In the case of PTCs, this regulatory mechanism is most likely the nonsense mediated decay (NMD), as previously reported in UCMD and BM patients. ${ }^{17,35}$ Nevertheless, we previously reported and confirm here that the homozygous nonsense mutation in 1 patient escaped the NMD process by skipping the mutation-bearing exon. ${ }^{26}$ Moreover, the presence of a heteroallelic missense mutation masked the impact of the PTC-causing mutation in 2 compound heterozygous patients.

Genotype-phenotype correlations have started to emerge recently, on limited groups of patients. ${ }^{16}$ The present study on a large cohort of patients also reveals several striking observations. In agreement with previous reports, ${ }^{21,30,36,37}$ all patients harboring recessive PTCcausing mutations in the $\mathrm{TH}$ region exhibit an earlysevere phenotype (ie, never acquired ambulation). However, 2 milder patients carry homozygous PTC-causing mutations located in $\mathrm{N}$-terminal domains. As mentioned before, for 1 of them we demonstrated that skipping of the mutated exon allowed the production of a shorter but functional protein. ${ }^{26}$ We have not been able to implicate the same mechanism in the other patient, who is still young ( 9 years old) and already shows scoliosis and respiratory problems, signs suggestive of a progressive course. Another mildly affected patient exhibited a PTCcausing mutation in the signal peptide, combined with a missense mutation, which likely modulates the deleterious effect of the PTC.

In addition, dominant splice-site mutations causing exon skipping as well as Gly-missense mutations were mostly observed in patients of the moderate-progressive category. Nevertheless, it is worth noting that skipping of exon 16 of $C O L 6 A 3$, recently described as the most common consequence of splice-site mutations, was detected also in patients with early-severe phenotypes, as observed by others. ${ }^{16,18}$ This severity is probably due to the proximity of the unique cysteine residue encoded by exon 17 of $C O L 6 A 3$, which is thought to be implicated in the disulfide bonds that assemble dimers and tetramers prior to secretion. ${ }^{38}$

However, it is important to note that similar types of mutations or mutations in the same domain of the protein do not necessary lead to comparable phenotype severity, because they have been identified in patients with variable clinical severity by us and others. ${ }^{11,15,39}$ Indeed, one cannot exclude that the numerous polymorphisms identified in these genes may contribute to the variability of the clinical phenotype. Further analysis of the segregation of these variants in affected individuals is necessary.

In conclusion, our data on this large cohort of patients with early onset ColVI myopathies reinforce previous reports relating to the variability of the clinical spectrum and the complexity of the diagnosis for this condition. Although the final diagnosis of ColVI myopathies may only 
be made on mutation identification in 1 of the COL6A genes, we propose that quantitative RT-PCR is helpful in accelerating the identification of the mutation-bearing gene. The frequency of dominant de novo mutations is very important to consider in the context of genetic counseling. Moreover, the identification of genotype-phenotype correlations may be useful in the design of future clinical trials.

\section{Acknowledgments}

This research was supported by funds from Institut National de la Santé et la Recherche Médicale (P.G and V.A), Association Française contre les Myopathies Grants $\mathrm{N}^{\circ} 13576$ (L.B), $\mathrm{N}^{\circ} 11595$ (P.G) and 13420 (V.A.), Association Institut de Myologie $N^{\circ} 13418$ (P.G.), GISInstitut des Maladies Rares, Assistance Publique-Hôpitaux de Paris (P.R., C.L.), and the ECOS SECYT French-Argentinean network A02S02 (A.L.T, P.G).

\section{Authorship}

L.B. and P.R. contributed equally. P.G. and V. A. contributed equally.

\section{Potential Conflicts of Interest}

Nothing to report.

\section{References}

1. Chu ML, Pan TC, Conway D, et al. Sequence analysis of alpha $1(\mathrm{VI})$ and alpha $2(\mathrm{VI})$ chains of human type $\mathrm{VI}$ collagen reveals internal triplication of globular domains similar to the $A$ domains of von Willebrand factor and two alpha $2(\mathrm{VI})$ chain variants that differ in the carboxy terminus. EMBO J 1989;8:1939-1946.

2. Chu ML, Zhang RZ, Pan TC, et al. Mosaic structure of globular domains in the human type VI collagen alpha 3 chain: similarity to von Willebrand factor, fibronectin, actin, salivary proteins and aprotinin type protease inhibitors. EMBO J 1990;9:385-393.

3. Engvall E, Hessle H, Klier G. Molecular assembly, secretion, and matrix deposition of type VI collagen. J Cell Biol 1986;102:703-710.

4. Allamand V, Merlini L, Bushby K. 166th ENMC International Workshop on Collagen type VI-Related Myopathies, 22-24 May 2009, Naarden, the Netherlands. Neuromuscul Disord 2010;20:346-354.

5. Lampe AK, Bushby KMD. Collagen VI related muscle disorders. J Med Genet 2005;42:673-685.

6. Nonaka I, Une Y, Ishihara $T$, et al. A clinical and histological study of Ullrich's disease (congenital atonic-sclerotic muscular dystrophy). Neuropediatrics 1981;12:197-208.

7. Ullrich O. Kongenitale, atonisch-sklerotische Muskeldystrophie, ein weiteres Typus der heredodegenerativen Erkrankungen des neuromuskulären Systems. Z Gesamte Neurol Psychiatr 1930;126: 171-201.

8. Bethlem J, Van Wijngaarden GK. Benign myopathy with autosomal dominant inheritance: a report of three pedigrees. Brain 1976;99:91-100.

9. De Visser M, van der Kooi AJ, Jobsis GJ. Bethlem myopathy. In: Engel AG, Franzini-Armstrong C, eds. Myology. New York, NY: McGraw-Hill, 2004;1135-1146.
10. Merlini L, Martoni E, Grumati $\mathrm{P}$, et al. Autosomal recessive myosclerosis myopathy is a collagen VI disorder. Neurology 2008;71: 1245-1253.

11. Scacheri PC, Gillanders EM, Subramony SH, et al. Novel mutations in collagen $\mathrm{VI}$ genes: expansion of the Bethlem myopathy phenotype. Neurology 2002;58:593-602.

12. Baker NL, Morgelin M, Peat R, et al. Dominant collagen VI mutations are a common cause of Ullrich congenital muscular dystrophy. Hum Mol Genet 2005;14:279-293.

13. Giusti B, Lucarini L, Pietroni $V$, et al. Dominant and recessive COL6A1 mutations in Ullrich scleroatonic muscular dystrophy. Ann Neurol 2005;58:400-410.

14. Jimenez-Mallebrera C, Maioli MA, Kim J, et al. A comparative analysis of collagen $\mathrm{VI}$ production in muscle, skin and fibroblasts from 14 Ullrich congenital muscular dystrophy patients with dominant and recessive COL6A mutations. Neuromuscul Disord 2006; 16:571-582.

15. Lampe AK, Dunn DM, von Niederhausern AC, et al. Automated genomic sequence analysis of the three collagen $\mathrm{VI}$ genes: applications to Ullrich congenital muscular dystrophy and Bethlem myopathy. J Med Genet 2005;42:108-120.

16. Lampe AK, Zou Y, Sudano D, et al. Exon skipping mutations in collagen $\mathrm{VI}$ are common and are predictive for severity and inheritance. Hum Mutat 2008;29:809-822.

17. Lucarini L, Giusti B, Zhang R-Z, et al. A homozygous COL6A2 intron mutation causes in-frame triple-helical deletion and nonsense-mediated mRNA decay in a patient with Ullrich congenital muscular dystrophy. Hum Genet 2005;117:460-466.

18. Okada M, Kawahara G, Noguchi S, et al. Primary collagen VI deficiency is the second most common congenital muscular dystrophy in Japan. Neurology 2007;69:1035-1042

19. Pace R, Peat R, Baker N, et al. Collagen VI glycine mutations: perturbed assembly and a spectrum of clinical severity. Ann Neurol 2008;64:294-303.

20. Pan T, Zhang R, Sudano D, et al. New molecular mechanism for Ullrich congenital muscular dystrophy: a heterozygous in-frame deletion in the COL6A1 gene causes a severe phenotype. Am J Hum Genet 2003;73:355-369.

21. Peat RA, Baker NL, Jones $\mathrm{KJ}$, et al. Variable penetrance of COL6A1 null mutations: implications for prenatal diagnosis and genetic counselling in Ullrich congenital muscular dystrophy families. Neuromuscul Disord 2007;17:547-557.

22. Pepe G, Lucarini L, Zhang RZ, et al. COL6A1 genomic deletions in Bethlem myopathy and Ullrich muscular dystrophy. Ann Neurol 2006;59:190-195.

23. Pepe G, Bertini E, Giusti B, et al. A novel de novo mutation in the triple helix of the COL6A3 gene in a two-generation Italian family affected by Bethlem myopathy. A diagnostic approach in the mutations' screening of type VI collagen. Neuromuscul Disord 1999;9:264-271.

24. Foley $A R, \mathrm{Hu} Y$, Zou $Y$, et al. Autosomal recessive inheritance of classic Bethlem myopathy. Neuromuscul Disord 2009;19:813-817.

25. Gualandi F, Urciuolo A, Martoni E, et al. Autosomal recessive Bethlem myopathy. Neurology 2009;73:1883-1891.

26. Demir E, Sabatelli P, Allamand V, et al. Mutations in COL6A3 cause severe and mild phenotypes of Ullrich congenital muscular dystrophy. Am J Hum Genet 2002;70:1446-1458.

27. Livak KJ, Schmittgen TD. Analysis of Relative Gene Expression Data Using Real-Time Quantitative PCR and the $2-\Delta \Delta C T$ Method. Methods 2001;25:402-408.

28. Demir E, Ferreiro A, Sabatelli P, et al. Collagen VI status and clinical severity in Ullrich congenital muscular dystrophy: phenotype analysis of 11 families linked to the COL6 loci. Neuropediatrics 2004;35:103-112. 
29. Quijano Roy S, Allamand V, Riahi N, et al. Predictive factors of severity and management of respiratory and orthopaedic complications in 16 Ullrich CMD patients. 12th International Congress of the World Muscle Society. Giardini Naxos- Taormina Mare, Messina, Sicily Italy. Neuromuscul Disord 2007;17:844.

30. Camacho Vanegas O, Bertini E, Zhang R-Z, et al. Ullrich scleroatonic muscular dystrophy is caused by recessive mutations in collagen type VI. Proc Natl Acad Sci U S A 2001;98: 7516-7521.

31. Pepe G, Bertini E, Bonaldo P, et al. Bethlem myopathy (BETHLEM) and Ullrich scleroatonic muscular dystrophy: 100th ENMC International Workshop, 23-24 November 2001, Naarden, the Netherlands. Neuromuscul Disord 2002;12:984-993.

32. Nadeau A, Kinali M, Main $M$, et al. Natural history of Ullrich congenital muscular dystrophy. Neurology 2009;73:25-31.

33. Lamandé $\mathrm{SR}$, Morgelin $\mathrm{M}$, Adams NE, et al. The C5 domain of the collagen $\mathrm{VI}$ alpha $3(\mathrm{VI})$ chain is critical for extracellular microfibril formation and is present in the extracellular matrix of cultured cells. J Biol Chem 2006;281;16607-16614.
34. Martoni E, Urciuolo A, Sabatelli $P$, et al. Identification and charac terization of novel collagen $\mathrm{VI}$ non-canonical splicing mutations causing Ullrich congenital muscular dystrophy. Hum Mutat 2009 ; 30:E662-E672.

35. Lamandé SR, Bateman JF, Hutchison W, et al. Reduced collagen $\mathrm{VI}$ causes Bethlem myopathy: a heterozygous COL6A1 nonsense mutation results in mRNA decay and functional haploinsufficiency. Hum Mol Genet 1998;7:981-989.

36. Higuchi I, Shiraishi T, Hashiguchi T, et al. Frameshift mutation in the collagen VI gene causes Ullrich's disease. Ann Neurol 2001;50:261-265.

37. Ishikawa H, Sugie K, Murayama K, et al. Ullrich disease: collagen $\mathrm{VI}$ deficiency: EM suggests a new basis for muscular weakness. Neurology 2002;59:920-923

38. Furthmayr $\mathrm{H}$, Wiedemann $\mathrm{H}$, Timpl $\mathrm{R}$, et al. Electron-microscopical approach to a structural model of intima collagen. Biochem $\mathrm{J}$ 1983;211:303-311

39. Lucioli S, Giusti B, Mercuri E, et al. Detection of common and private mutations in the COL6A1 gene of patients with Bethlem myopathy. Neurology 2005;64:1931-1937. 